\title{
1 Insect egg-killing: a new front on the evolutionary arms-race between Brassicaceae plants and
}

\section{Pierid butterflies}

3

4 Eddie Griese ${ }^{1,2}$, Lotte Caarls ${ }^{1}$, Setareh Mohammadin ${ }^{1}$, Niccolò Bassetti ${ }^{1}$, Gabriella

5 Bukovinszkine'Kiss ${ }^{1,3}$, Floris C. Breman ${ }^{1}$, Erik H. Poelman ${ }^{2}$, Rieta Gols ${ }^{2}$, M. Eric Schranz ${ }^{1}$ and

6 Nina E. Fatouros ${ }^{1, *}$

7

$8 \quad{ }^{1}$ Biosystematics Group, Wageningen University, Wageningen, The Netherlands

$9 \quad{ }^{2}$ Laboratory of Entomology, Wageningen University, Wageningen, The Netherlands

$10{ }^{3}$ Current address: Laboratory of Genetics, Wageningen University, Wageningen, The Netherlands

11 *corresponding author: nina.fatouros@wur.nl

\section{Abstract}

14 Evolutionary arms-races between plants and herbivores have been proposed to generate key

15 innovations that can drive diversification of the interacting species. Recent studies reveal that plant traits that target herbivore insect eggs are widespread throughout the plant kingdom. Within the Brassicaceae family, some plants express a hypersensitive response (HR)-like necrosis underneath the eggs of specialist cabbage white butterflies (Pieridae) that leads to eggs desiccating or dropping of the leaf. Here, we studied the evolutionary basis of this trait, its egg-killing effect on and elicitation by specialist butterflies, by screening 31 Brassicaceae species and nine Pieridae species.

21 We show that induction of HR-like necrosis by pierid egg deposition is clade-specific in the

22 economically important Brassiceae tribe (Brassica crops and close-relatives) and in the firstbranching genus Aethionema. The necrosis is elicited only by pierid butterflies that feed on 
26 Rhamnaceae (Gonepteryx rhamni) or Fabaceae (Colias spp.) however, did not elicit such a leaf

27 necrosis. Finally, eggs of Aglais io, a species of the sister group Nymphalidae, did not elicit any

28 visible response. Counter-adaptations to HR-like necrosis might have evolved by insect deposition

29 of eggs in clusters or on inflorescences. Our findings suggest that the plants' egg-killing trait is a

30 new front on the evolutionary arms-race between Brassicaceae and pierid butterflies beyond the

31 well-studied chemical defence traits against caterpillars.

32

33 Key words: induced plant defences, counter adaptation, coevolution, plant-insect interaction, egg

34 deposition, hypersensitive response

35

36

37 


\section{Introduction}

40 The biodiversity on earth is shaped by numerous factors including inter-organismal interactions that can result in coevolution of adaptive traits. For example, the coevolutionary interactions between plants and insects as described by Ehrlich and Raven ${ }^{1}$ has driven the diversification of plant defensive metabolites ${ }^{2,3}$. In turn, specialist herbivores have evolved detoxification mechanisms, which allow them to feed on their host plants despite these toxic metabolites ${ }^{4,5}$, e.g. monarch butterflies can feed on cardenolide-containing milkweeds ${ }^{6,7}$, and Pieridae and Plutella caterpillars on glucosinolate-containing Brassicaceae ${ }^{8-10}$.

The role of plant defences against herbivore eggs has been understudied and underappreciated, especially in a coevolutionary perspective between herbivores and plants. The majority of studies on plant-insect interactions have focused on the feeding life stages of herbivorous insects. Yet, plants can already perceive and respond physiologically to the presence of herbivore eggs before they hatch ${ }^{11}$. The evolution of plant defences against insect eggs is an important first line of defence. In almost half of the $\sim 400.000$ known herbivorous insects, especially in case of lepidopteran and sawfly species, eggs may be the first life stage to come into contact with the targeted host plant. Every insect egg being detected and killed, is one less herbivorous larva or adult insect feeding on the plant in the near future.

Different types of plant defences against insect eggs have been reported in more than thirty plant species including gymnosperms and angiosperms (both monocots and eudicots) ${ }^{12}$. In response to 60 insect egg deposition, plants can produce ovicidal substances ${ }^{13}$, form neoplasms ${ }^{14,15}$ or express a 61 hypersensitive response (HR)-like necrosis beneath the eggs ${ }^{15-19}$. Specifically, HR-like necrosis as 62 an egg-killing defence leading to eggs desiccating and/or falling off the leaf. It has so far been 
63 observed in plants of the Pinaceae ${ }^{20}$, Poaceae $^{21}$, Fabaceae $^{22}$, Solanaceae $^{15,16}$ and Brassicaceae $e^{17-19,23}$

64 families. However, the phylogenetic occurrence of the egg-killing trait across these plant families

65 and the phylogenetic co-occurrence in the reciprocal insect pest-clade has yet to be investigated in a similar manner to recent studies of plants and their insect herbivores such as the Brassicaceae plants and Pieridae caterpillars.

Sequence-based phylogenetic analysis ${ }^{24-26}$ has established that the Brassicaceae family is split into a core clade containing 3680 species, sub-divided into three major lineages, and a smaller sister clade containing only the genus Aethionema (61 species $^{27,28}$ ). The model plant Arabidopsis thaliana is a representative of Lineage I and the Brassica crop plants are representatives of Lineage II. Lineage III is a smaller group mostly restricted to Asia and lacking a model or crop species. Cleomaceae is the sister family of the Brassicaceae ${ }^{29}$. Within the Brassicaceae, defences against feeding herbivores and the genetic basis of this defence have intensively been studied ${ }^{30-33}$. Aliphatic glucosinolates evolved as defensive compounds near or at the origin of the Brassicales clade and became more diverse and complex with plant species radiation. While these compounds play an important role in defending the plants against herbivory, many feeding insects have specialized and evolved effective glucosinolate detoxification and/or excretion mechanisms ${ }^{8,34-36}$.

The Pieridae (whites and sulphurs), containing some 17000 species today, use two major host plants belonging to the Fabales (Fabaceae) and Brassicales (Brassicaceae, Resedaceae, Capparaceae and

83 Cleomaceae); species in some clades also shifted to Rosales (Rhamnacea, Rosaceae) or

84 Santalales ${ }^{9,37}$. Recent phylogenetic reconstruction of the Pieridae indicate that the ancestral host 85 appears to be Fabaceae with multiple independent shifts to other orders. While the Dismorphiinae 86 and nearly all Coliadinae are Fabales feeders, the sister to the Coliadinae, Pierinae, primary feed on 87 Brassicales $^{38}$. The latter thus represent a single origin of glucosinolates feeding ${ }^{9}$. Shortly after the 
initial evolution of the order Brassicales, some ancestral Pierinae were able to evolve nitrilespecifier proteins (NSPs) that detoxify glucosinolates. This enabled a host shift from their prior Fabaceae hosts to the Brassicales roughly 80 million years ago ${ }^{9,37}$. Similarly, the evolution of glucosinolate sulfatase in Plutella xylostella allowed the caterpillar of these moths to feed on Brassicaceae $^{8}$. It has been shown that speciation-rate shifts, as well as genome-duplication events with gene birth-death dynamics, occurred in both Brassicales and Pieridae, usually following a key defence (glucosinolates) or counter-defence (NSPs and sulfatase) invention in one of the coevolutionary partners ${ }^{37}$. To pinpoint the evolution of transitions and innovations, it is necessary to have investigate the trait(s) of interest in a proper phylogenetic context. Defence responses targeting eggs might add a new layer of traits evolved in response to herbivore specialization. Egg-killing responses could then be understood as a first-line-of-defence on top of the later acting glucosinolate defence system.

Eggs of the specialist herbivore Pieris brassicae induce HR-like necrosis in the crop plants Brassica rapa, B. napus and Raphanus sativus ${ }^{12,39}$. However, egg-induced responses have mainly been studied in the black mustard Brassica nigra and the model plant A. thaliana. On A. thaliana egg deposition induces a localized cell death response and higher expression of defence genes resembling HR against pathogens, but a visible necrosis is not expressed and egg-killing never been shown $^{40,41}$. Egg-killing due to a strong necrosis has been shown for the black mustard B. nigra.

Within B. nigra, HR-like necrosis shows high intraspecific variation. Several B. nigra accessions were tested with regard to their ability to express HR-like necrosis in response to egg depositions, with some accessions being more likely to express this trait than others ${ }^{17,18,23}$.

The current study explores whether egg-killing necrosis evolved as a specific response to pierid egg deposition in a subset of Brassicaceae. So far, no large-scale screening has been done within the 
113 family to determine how common the egg-killing necrosis is expressed within the family.

114 Furthermore, no effort has ever been made to map the phylogenetic history of any egg defence trait

115 for any plant family. Doing so would be a first necessary step to show an adaptive response to egg

116 deposition. For this study we first established that egg wash generated from eggs of P. brassicae

117 butterflies and egg deposition on plants yielded a similar plant response on $B$. nigra plants. We then

118 used a representative collection of species in the Brassicaceae (mainly lineage I and II) and three

119 species in the Cleomaceae to investigate the phylogenetic occurrence of egg-killing necrosis across

120 the family. Furthermore, we explored the reciprocal phylogenetic co-occurrence in the Pieridae

121 clade and related species. We compared elicitation of HR-like response by egg deposition and egg

122 wash of three other Pieris butterflies (Pierinae) as well as by three relatives, Anthocharis

123 cardamines (Pierinae) feeding on Cardamine plants of Lineage I, Colias spp. (Coliadinae) feeding

124 on Fabaceae and Gonopteryx rhamni (Coliadinae) feeding on Rhamnus plants belonging to

125 Rhamnaceae. As an outgroup, we used the butterfly Aglais io (Lepidoptera: Nymphalidae) that

126 feeds on Urtica plants (Urticaceae). We addressed the following questions: (i) Is HR-like necrosis

127 induced in a clade-specific manner within the Brassicaceae? (ii) Is the observed necrosis lowering

128 egg survival under greenhouse and field conditions? (iii) Is elicitation of HR-like necrosis by eggs

129 specific to a particular clade of butterfly species (e.g. genus, subfamily or family) and/or specific to

130 species that co-evolved with the Brassicaceae?

132 Material and Methods

133 Plants and insects

134 For our study, we obtained seeds of twenty-eight Brassicaceae and three Cleomaceae species from

135 various sources. The selected plants represent the major lineages in each family. For each plant

136 species, between one and eleven accessions were obtained (Table S1). Per accession, between three

137 and seventeen plants were phenotyped across members of the two families. Two accessions of $B$. 
nigra (SF48, SF19) were used to assess elicitation of the HR-like necrosis by different butterfly

species. Finally, egg-killing was tested for four responsive plant species with the same number of genotypes per species. In preliminary trials, plant species with unknown developmental times were

142 similar life stages, i.e. vegetative growth, and sizes if possible. Therefore, plants were between three

143 and six weeks old when being treated with butterfly eggs or egg wash.

144 For phenotyping the Brassicaceae we used the wash of Pieris brassicae eggs. To assess induction of

145 HR-like necrosis on B. nigra plants, we used egg deposition from two populations of $P$. brassicae,

146 P. napi L. and P. rapae L. and one population of P. mannii Mayer (Table S2). Furthermore, we

147 tested egg wash from three populations of A. cardamines L., and one population of G. rhamni L.

148 and A. io L. (Lepidopera: Nymphalidae) (Table S2). Finally, survival was measured for eggs of $P$.

149 brassicae, $P$. napi and P. rapae.

150 Pieris brassicae, $P$. napi and P. rapae were reared on Brassica oleracea var. gemmifera cv. Cyrus

151 in a greenhouse compartment $\left(21 \pm 4^{\circ} \mathrm{C}, 60-80 \%\right.$ RH, LD 16: 8). Pieris mannii was reared in the

152 same greenhouse, but instead on flowering Iberis spp. plants. One population of $A$. cardamines was

153 obtained from a butterfly farm Farma Motyli Zielona Dolina (Babidół, Poland) as hibernating

154 pupae. Hibernation was broken by storing the pupae at $4{ }^{\circ} \mathrm{C}$ in a cold storage room for five months

155 and another month outdoors. After hibernation, the butterflies were kept in a greenhouse

156 compartment $\left(18 \pm 2^{\circ} \mathrm{C}, 50-60 \%\right.$ RH, LD 16: 8) with flowering Cardamine hirsuta and Sisymbrium

157 irio plants to obtain eggs. Aglais io butterflies were kept in cages outside (May to June 2018) with

158 cuttings of Urtica sp. plants on which they oviposited. Eggs and/or adults of A. cardamines, Colias

159 spp. and G. rhamni were also collected outdoors (for locations see table S2); adults were released

160 again when sufficient egg depositions were obtained. Pieris brassicae and A. io both lay egg

161 clutches, P. napi sometimes lays eggs in small groups, while A. cardamines, G. rhamni, P. mannii

162 and $P$. rapae lay single eggs. 
164 Egg wash preparation

165 Wash from $P$. brassicae eggs was made by fostering females to oviposit on filter paper by pinning

166 the paper to the underside of leaves of B. oleracea (Fig. 1a). Within 24 hours after oviposition, the

167 filter paper with the eggs was cut and placed into a $15 \mathrm{ml}$ Falcon tube with purified water

168 (purification system from Millipore Company) at a concentration of 400 eggs per ml. The eggs were

169 left overnight at room temperature. The next morning the supernatant was pipetted off and stored at

$170-20{ }^{\circ} \mathrm{C}$. Before using the egg wash, Tween 20 was added at a $0.005 \%$ concentration. The addition of

171 Tween20 was necessary to lower the surface tension of the water droplets, therefore improving the

172 distribution of the egg wash on the waxy leaf surface of some plant species.

173 

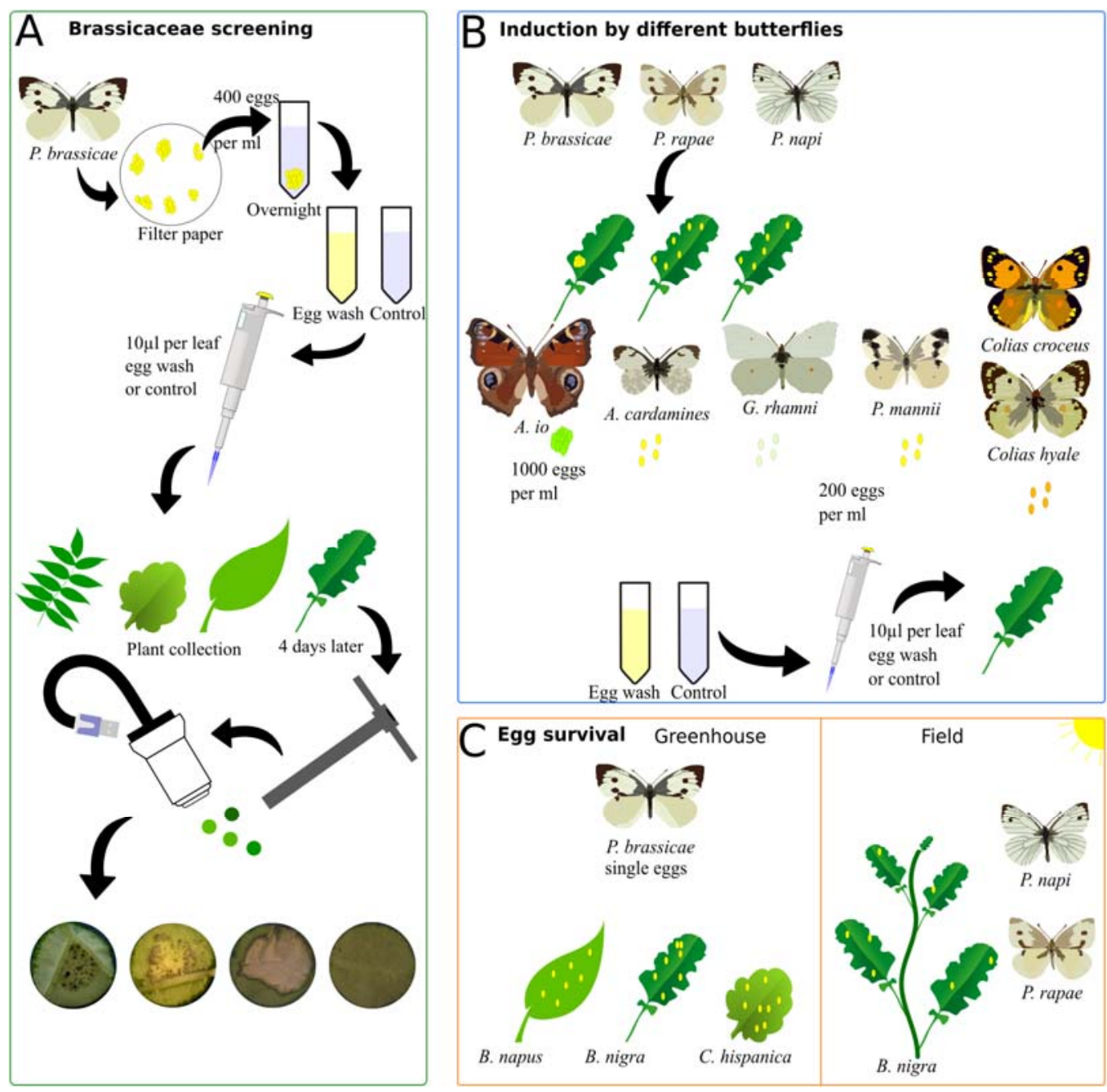

174 Figure 1: Scheme for plant treatments and phenotyping of HR-like necrosis. A) Production and use

175 of wash from $P$. brassicae egg clusters for a screening of 31 plant species, each of which consisted

176 of 1 to 10 plant accessions. B) Use of eggs or egg wash from different butterfly species to determine

177 which species elicits a necrosis in B. nigra accessions. C) Use of singly laid P. brassicae eggs to

178 determine the egg-killing effect of HR-like necrosis on B. napus, B. nigra and C. hispanica

179 accessions. From the field $P$. napi and $P$. rapae eggs were collected from $B$. nigra and hatching

180 (survival) observed.

182 Wash from A. io, G. rhamni and A. cardamines eggs was made by removing eggs from leaves of

183 Urtica sp. (A. io) or Rhamnus sp. (G. rhamni) and floral inflorescences of C. hirsuta or S. irio (A. 
184 cardamines). These eggs were immersed in pure water (A. io) or $20 \mathrm{mM}$ 2-( $N$-morpholino) ethane-

185 sulfonic acid (MES) buffer (A. cardamines) and left overnight. We chose a concentration of 1000

186 eggs per $\mathrm{ml}$ for $A$. io, as egg size is lower than of Pierini eggs (compare database on egg size from

187 more than 10.000 insect species: https://shchurch.github.io/dataviz/index.html). As controls, clean

188 Urtica sp. leaves for A. io, a mixture of C. hirsuta and S. irio inflorescence stems for A. cardamines,

189 clean leaves of Rhamnus frangula L. for G. rhamni, and inflorescence stems of Iberis spp. For $P$.

190 mannii were washed in the same manner. Eggs and leaves were kept in the solution overnight, after

191 which the supernatant without eggs was pipetted off and stored at $-20{ }^{\circ} \mathrm{C}$. As these egg washes were

192 tested on B. nigra plants, no Tween20 was added to the washes.

193

194 Phenotyping of HR-like necrosis of Brassicales plants

195 Experiments were carried out in a greenhouse compartment to standardize plant-growth conditions

196 (22-27 ${ }^{\circ} \mathrm{C}, \mathrm{Rh}: 50-90 \%$, L:D: 16:8). For the screening of twenty-eight Brassicaceae and three

197 Cleomaceae plant species, $5 \mu \mathrm{l}$ of $P$. brassicae egg wash was pipetted on a fully mature leaf (the

198 third or fourth leaf from the top) of each plant. Another fully matured leaf (the third or fourth from

199 the top) received pure water with Tween20 as a control. After four days, leaf disks were harvested

200 of the area where egg wash had been applied using a cork borer $(1 \mathrm{~cm})$ and put in a rectangular Petri

201 dish with wet blue filter paper. Pictures were taken using a Dino-Lite digital microscope (AnMo

202 Electronics Corporation). These pictures were visually scored for expression of HR-like necrosis

203 (Fig. 1a).

204

205 Testing for elicitation of HR-like necrosis by diverse Pieridae species

206 Female butterflies of $P$. brassicae (2 populations), $P$. napi and $P$. rapae (2 populations) were

207 allowed to lay between five to ten eggs on two different B. nigra accessions (SF19 and SF48)

208 (Supplementary Table 1). Accession SF19 is known as a low responder with respect to egg HR-like 

necrosis and SF48 as a strong responder ${ }^{18}$. Anthocharis cardamines, Colias sp. and G. rhamni egg wash was pipetted on both B. nigra accessions (Supplementary Table 1). The nymphalid Peacock butterfly A. io was used as an outgroup. Eggs laid on Urtica leaves were collected and an egg wash made as well as a control wash made from Urtica leaves and pipetted on plants of the same B. nigra accessions. Between 17 and 40 plant replicates per B. nigra accession were used for each butterfly population (Fig. 1b). After four days, HR-like necrosis was scored using a slightly adapted scoring system previously described by Griese et al. ${ }^{18}$. For this scoring system a number between 0 (no response) and 4 (very strong response on both sites of the leaf) is assigned to the observed necrosis.

\section{Pieris brassicae egg survival on HR-like expressing plants}

Experiments were done in greenhouse conditions $\left(21 \pm 5^{\circ} \mathrm{C}, \mathrm{Rh}: 45-70 \%\right.$, L16 : D8). HR-like necrosis has been shown to have weaker effects on egg-survival under greenhouse conditions than under natural conditions ${ }^{17,18}$. Pieris brassicae females were manipulated to lay five to fifteen separated eggs (not touching each other) on all lines of B. napus, B. nigra and C. hispanica used in the screening of Brassicaceae species. Previous studies revealed that $P$. brassicae egg survival was only affected when eggs were laid singly, not touching each other ${ }^{18}$. The oviposition of separated eggs was accomplished by observing the females and taking them off the leaf after they laid one egg. After this, the females were put on a different spot of the same leaf. The eggs were left on the plant and four days after oviposition HR-like necrosis was scored as present or absent. After five days, survival of eggs was noted by counting the number of hatched caterpillars (Fig. 1c).

\section{Pieris brassicae egg survival assessed by field survey}

A survey was conducted to record survival of Pieris eggs on individual B. nigra plants in a natural population (compare Fatouros, et al. ${ }^{17}$ ). The survey was conducted at an established B. nigra patch along the River Rhine in Wageningen (Steenfabriek), The Netherlands (coordinates: $51.96^{\circ} \mathrm{N}$, 
$2345.68^{\circ} \mathrm{E}$ ) in one season and butterfly generation (August-September 2017). The total area

235 monitored was approximately $100 \mathrm{~m}^{2}$ consisting of $\sim 1000$ plants. Plants were monitored for eggs at

236 the edges of a patch or on isolated growing plants So that not all 1000 plants were monitored.

237 Eggs were collected on leaves and checked for the presence of a HR-like necrotic zone on the leaf.

238 After collection, eggs were kept in a climate chamber $\left(25 \pm 1^{\circ} \mathrm{C}, 50-70 \% \mathrm{RH}, \mathrm{L} 16\right.$ : D8 $)$ until

239 caterpillars emerged. All hatched and dead eggs were recorded (Fig. 1c).

Phylogenetic analysis of Brassicales and Pieridae species

242 We used a consensus tree to place our tested Brassicales species according to the species (or genera)

243 reported by two recent studies ${ }^{25,26}$. Both studies analyse representatives of the three distinct linages

244 of the core Brassicaceae clade and the first-branching Aethionema and the outgroup Cleomaceae.

245 We used the established three-linage classification when planning and conducting our experiments.

246 As some species and genera were not present in either study, we established their relationships with

247 other included species by calculating our own phylogenetic tree using DNA sequences of two

248 chloroplast markers ( $r b c L$ and $m a t K)$ and one nuclear genome marker (ITS2). The sequences were

249 obtained from the BOLD system website (ID numbers see Supplementary Table 3$)^{42}$. The

250 phylogenetic tree was inferred under maximum likelihood using RaxML v 8.2.4 (GTR+GAMMA,

251 random seed and 1000 bootstrap pseudo-replicates) on the CIPRES science gateway ${ }^{43,44}$. The three

252 Cleomaceae species were used as outgroups for the phylogenetic tree.

253 The phylogenetic tree of the butterfly species was created using the mitochondrial COI gene and the

254 nuclear EFla (Supplementary Table 4). The phylogenetic tree was inferred using maximum

255 likelihood through the IQ TREE website ${ }^{45-47}$. The models selected here for each of the partitions

256 were GTR+F+I+G4:part1, TIM2e+G4:part2, random seed and 1000 ultrafast bootstrap pseudo-

257 replicates. We verified that each clade of butterflies in the tree contained more species than were

258 used in our test to improve separation. Plutella xylostella L. was used as an outgroup. The 
phylogeny showed support for splits within the Pieridae family and the genera were well supported.

260 The phylogeny is very similar to a more extensive study with more species that used two more

261 markers, wingless and $28 S^{48}$.

262 A Bayesian approach was also performed for phylogenetic inference of the butterflies using the

263 program MrBayes version $3.2^{49}$ on the same dataset using as priors the parameters from the models

264 selected by IQ TREE and using the same partition of the data. Four simultaneous chains (one cold,

265 three heated) were run for ten million generations, and trees were sampled every 1,000 generations.

266 To check the convergence and stability of the parameter estimates and to determine the burn-in

267 value, Tracer v1.5 $5^{50}$ was used to explore the log files. Initial trees generated in the burn-in phase

268 (i.e., before establishing stable estimates of parameters) were discarded (burn-in value $=2500,25 \%$

269 of the trees). The remaining trees were used to estimate tree topology, branch lengths, and

270 substitution parameters. The phylogenetic relationships inferred from this bayesian approach were

271 congruent with the ML tree obtained from the analysis above.

272

\section{Statistical analysis}

274 To test for statistical significance, R version 3.3.2 "Sincere Pumpkin Patch" ${ }^{51}$ was used. For the

275 screening of plant accessions, $\chi^{2}$-tests were used to determine which plant species/genotypes

276 significantly expressed HR-like necrosis after egg wash treatment compared to the control

277 treatment. The contingency tables for the $\chi^{2}$-tests consisted of the number of egg wash-treated

278 leaves expressing HR-like necrosis, the number of egg wash-treated leaves not expressing HR-like

279 necrosis, the number of control wash-treated leaves expressing HR-like necrosis and the number of

280 control wash-treated leaves not expressing HR-like necrosis. With this set-up, all plant accessions

281 from each plant species were tested independently.

282 Egg survival was analysed using binomial generalized linear models (GLMs) in which first all

283 variables (plant species, flowering state, HR expression and all interactions between the factors) 
284

285

286

287

288

289

290

291

292

293

294

295

296

297

298

299

300

301

302

303

304

305

306

307

308

were used and then based on Akaike information criterions (AICs) removed to simplify the model (plant species, HR expression and interaction). After this, EMMEANS test or Mann-Whitney-U tests were performed as post-hoc tests. Differences in induction of HR-like necrosis by different butterflies were tested using binomial GLMs and, to test differences in strength, GLMs with Poisson distribution Dunn tests with Bonferroni-Holm correction were used as post-hoc tests.

\section{Results}

Establishing egg wash as an alternative treatment for natural egg deposition

Not all tested butterfly species naturally deposit eggs on (all) brassicaceous species. In order to be able to test eggs of those species and screen a large number of brassicaceous species efficiently, we developed a standard method to wash eggs and treat plants with egg wash. We first compared the effect of eggs and egg wash on B. nigra, and scored symptoms induced by oviposition or egg wash, scoring a number between 0 (no response) and 4 (very strong response). The accession SF48 responded with a score between 1-4 in all plants (Supplementary Figure 1). There was no statistical difference between class of symptoms induced by eggs or egg wash (GLM: $\chi^{2}=1.43, \mathrm{df}=1, P=$ 0.232), and so we concluded that we could use egg wash to test the effect on all species.

\section{Origin of HR-like necrosis in the core Brassicaceae, Aethionema and Cleomaceae}

Of all thirty-one species tested, five species responded significantly with HR-like necrosis to $P$. brassicae egg wash. This included species of the genus Aethionema and of the tribe Brassiceae (Fig. 2). In the tribe Brassiceae, egg wash treatment significantly enhanced expression of HR-like necrosis in specific accessions of four species: B. napus (25-86\%), B. nigra (63-83\%), B. oleracea (20-40\%) and C. hispanica (0-86\%) (Supplementary Table 5). There was no significant enhanced HR-like necrosis after egg wash treatment for all other tested plant species tested compared to control leaves. Necrosis was expressed in single plants of some accessions in lineage I and III (0 
309 and 29\%) (Fig. 2, Supplementary Table 5). HR-like necrosis of Aethionema arabicum varied among

310 the tested accessions between 0 and $60 \%$ (Supplementary Table 5). In some cases, e.g. for

311 Aethionema carneum, plants responded with HR-like necrosis to egg wash, however, due to the low

312 number of replicates (A. carneum: three plants) difference between control and egg wash treatment

313 was not significant (Supplementary Table 5). For Lunaria annua, up to $40 \%$ expressed HR-like

314 necrosis, but for this plant species only few replicates were tested, making it impossible to test for

315 significant differences (Supplementary Table 5). 


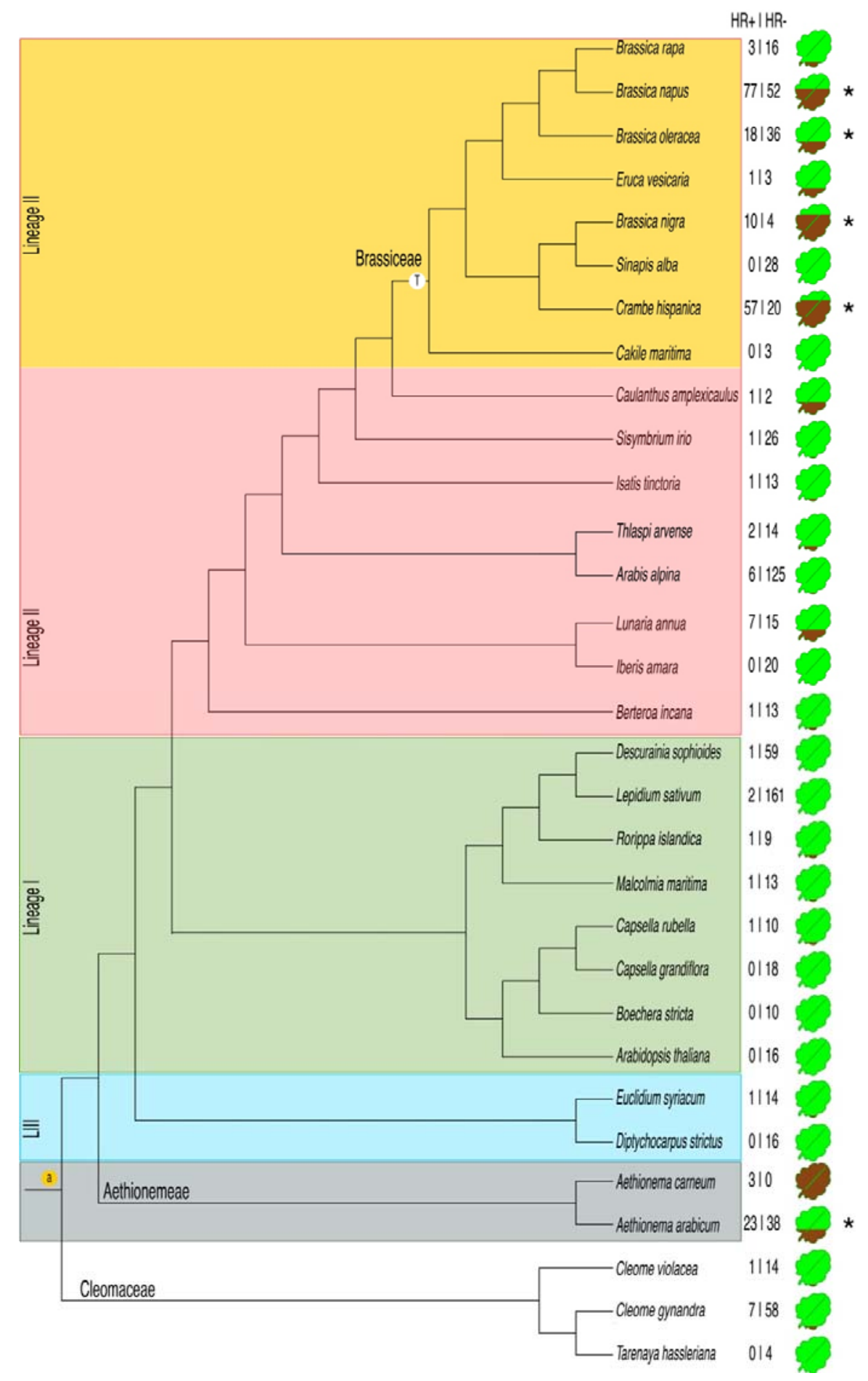

317 Figure 2: Phylogenetic tree of all plant species treated with $P$. brassicae egg wash and the

318 resulting- fraction of necrosis after 4 days. Consensus phylogeny based on literature and our own

319 analysis of 3 marker genes: rbcL and matK and one nuclear genome marker: ITS2 used. The brown 
320 part of the leaf shape represents the percentage of tested plants per plant species responding to egg

321 wash with necrosis. Asterisks indicate that at least one plant accession within the species showed

322 significantly more HR-like necrosis on leaves treated with egg wash than on control treated leaves

$323\left(\chi^{2}\right.$-tests, $\left.\mathrm{P}<0.05\right)$. Phylogenetic clades are coloured differently in the tree. The whole genome

324 duplication WGD (a) and genome triplication (T) the Brassiceae tribe specific events are marked in

325 the tree.

326

327 Elicitation of HR-like necrosis by different butterfly species correlated with phylogenetic signal

328 Egg deposition by all Pieris spp. and egg wash of A. cardamines elicited a HR-like necrosis on both

329 tested B. nigra accessions; the low responding SF19 and as the strong responding SF48. Egg wash

330 of G. rhamni and Colias spp. did not elicit a HR-like necrosis. Notably, egg wash of both species

331 induced the formation of chlorotic tissue (Fig. 3). Egg wash from A. io neither elicited a chlorosis

332 nor HR-like necrosis on either B. nigra accession (Table 1). When several populations were

333 available for butterfly species, all populations elicited HR-like necrosis in similar frequency (GLM:

$\left.334 \chi^{2}=1.36, \mathrm{df}=3, P=0.71\right)$ and severity $\left(\mathrm{GLM}: \chi^{2}=2.60, \mathrm{df}=3, P=0.46\right)$. 

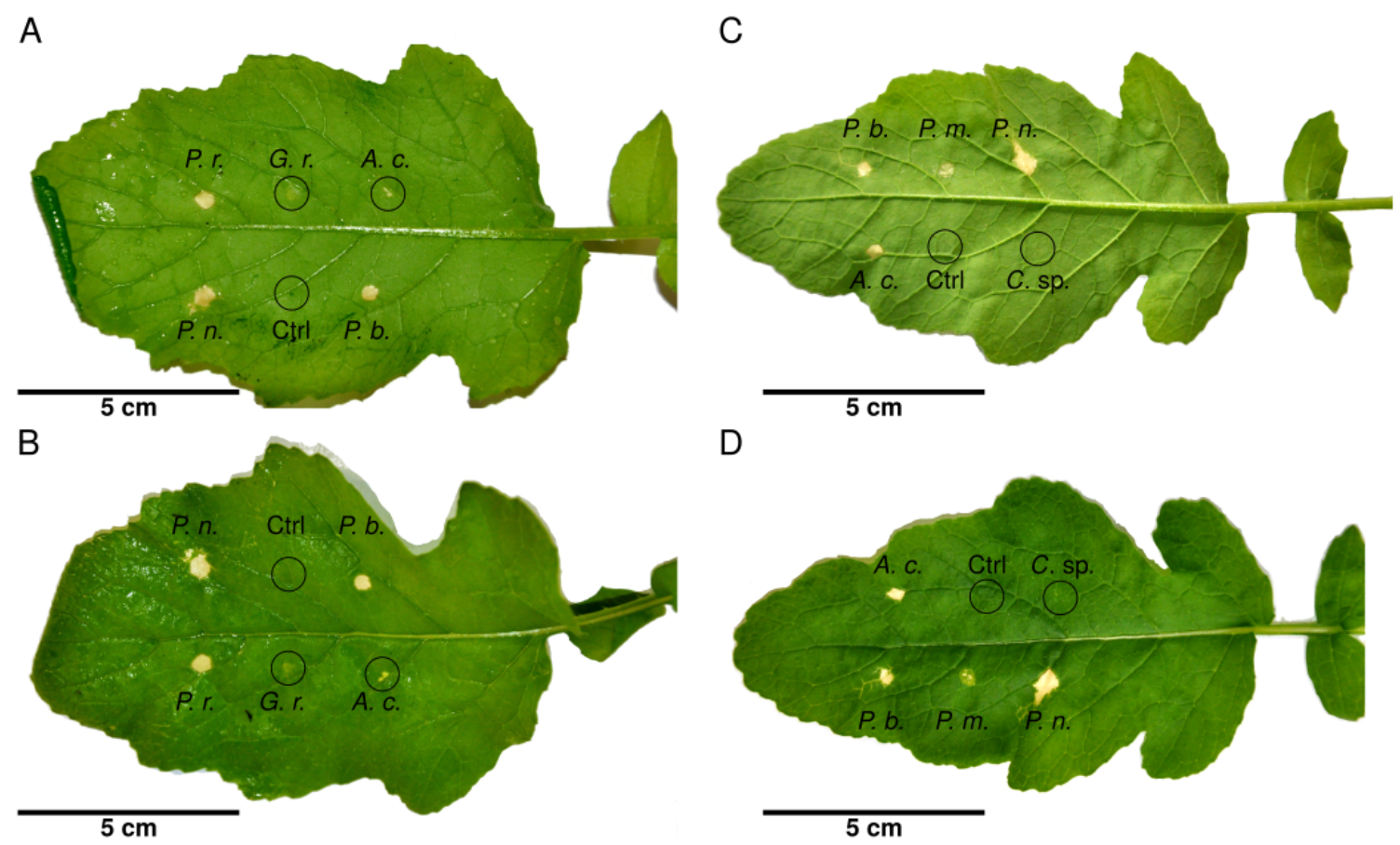

337 Figure 3: Leaves from B. nigra treated with egg wash of different butterfly species and controls

338 inducing or not a HR-like necrosis. Pieris brassicae (P. b.), P. mannii, (P. m.), P. napi (P. n.), and

339 P. rapae (P. r.) and Anthocharis cardamines (A. c.) induce a strong HR-like necrosis. Egg wash of

340 G. rhamni (G. r.) and Colias sp. (C. sp.) induces a very faint response resembling a chlorosis and

341 does not fit into the established scoring system (faintness indicates 1, but showing up on both sides

342 of the leaf indicates 2). The control (buffer without eggs) does not elicit a HR-like necrosis. All egg

343 washes had the same concentration (200 eggs per ml) and amount applied onto the leaf ( $5 \mu \mathrm{l})$. Two

344 leaves were needed as not all egg washes were available at the same time. A) and C) Abaxial side of

345 the leaf where the egg washes were applied onto. B) and D) Adaxial side of the leaf showing how

346 strong the HR-like response is on the side which was not treated with egg wash.

347 Table 1: HR- like necrosis (score ranging from 0 to 4) expressed by B. nigra plants elicited by

348 different butterfly species. HR-plants did not express HR-like necrosis, while HR+ plants did.

349 Different letters indicate significant differences (different when $\mathrm{P}<0.025$ ) between butterfly

350 species, Dunn-test, Bonferroni Holm corrected. 


\begin{tabular}{l|lll|l} 
Butterfly species & HR score (SE) & $\begin{array}{l}\text { Plants } \\
\text { HR+ }\end{array}$ & $\begin{array}{l}\text { Plants } \\
\text { HR- }\end{array}$ & HR fraction (SE) \\
\hline $\begin{array}{l}\text { Anthocharis } \\
\text { cardamines }\end{array}$ & $1.63(0.10) \mathrm{a}$ & 61 & 5 & $0.92(0.03) \mathrm{a}$ \\
\hline $\begin{array}{l}\text { Aglais } \text { io } \\
\text { Colias spp. }\end{array}$ & $0(0) \mathrm{b}$ & 0 & 40 & $0(0) \mathrm{b}$ \\
\hline Gonepteryx rhamni & $0.67(0.10) \mathrm{ab}$ & 4 & 5 & $0.56(0.18) \mathrm{a}$ \\
\hline Pieris brassicae & $1.11(0.33) \mathrm{a}$ & 8 & 10 & $0.44(0.12) \mathrm{c}$ \\
\hline Pieris mannii & $1.69(0.13) \mathrm{a}$ & 53 & 12 & $0.82(0.05) \mathrm{a}$ \\
\hline Pieris napi & $2.14(0.40) \mathrm{ac}$ & 6 & 1 & $0.86(0.14) \mathrm{ac}$ \\
\hline Pieris rapae & $2.46(0.16) \mathrm{c}$ & 33 & 4 & $0.89(0.05) \mathrm{a}$ \\
\hline
\end{tabular}

353 Eggs of all brassicaceous specialists, Pieris brassicae, P. napi, P. rapae and A. cardamines induced

354 an equally high fraction of HR-like necrosis in B. nigra (Supplementary Tables 1 and 6). Pieris napi

355 elicited a significantly stronger HR-like necrosis (2.46 \pm 0.16$)$ compared to all other butterfly

356 species (Supplementary Tables 1 and 7). The fraction and severity of chlorotic tissue formation

357 elicited by Colias spp. and G. rhamni was generally lower than HR-like necrosis by the eggs of

358 Pieris spp and A. cardamines $(0.44 \pm 0.12 ; 1.11 \pm 0.33$ respectively) (Table 1 and Supplementary

359 Tables 6-7). When we plotted the fraction of HR-like necrosis and its severity per butterfly species

360 on our phylogeny, the likelihood and severity of HR-like necrosis is stronger in butterfly species

361 that are the more closely related to Pieris sp. (Fig. 4). Thus, all tested Pieridae elicited an egg

362 response while the nymphalid butterfly A. io of the sister group never did. 


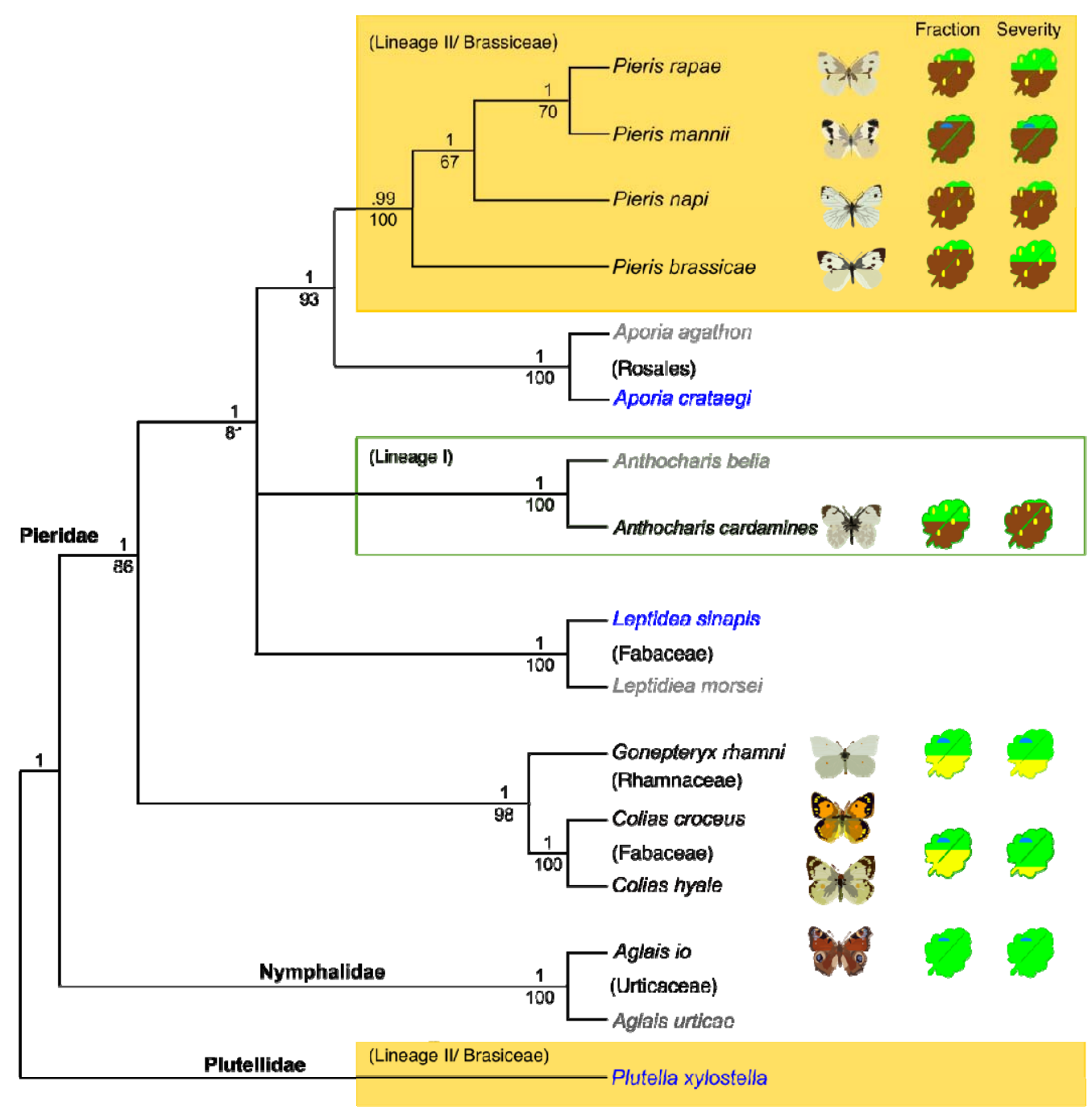

364 Figure 4: Phylogeny of a subset of Pieridae and elicitation of HR-like necrosis on $B$. nigra leaves

365 by pierid egg wash or eggs. The phylogeny is based on the maximum likelihood and Bayesian

366 posterior probability analysis of the nuclear marker EF1 $\alpha$ and mitochondrial maker COI subunit 1.

367 As outgroups, the nymphalid Aglais io and the plutellid moth Plutella xylostella were chosen. The

368 pictograms of leaves on the right of the cladogram represent the fraction of HR-like necrosis

369 elicitation (left) and severity of HR-like necrosis expressed (right). The average fraction (between 0

370 and 1) and severity (between 0 and 4) elicited by either eggs or egg wash is represented by the

371 brown part of the leaf, while the yellowing in the leaves represents a different type of response

372 (chlorosis). The phylogenetic tree consists of species used in the experiments (black), species that 
373 would answer open questions when tested (blue) and species added to more fully represent the

374 phylogenetic tree (grey). Coloured boxes indicate the Brassicaceae linage which the butterflies use

375 as main host plants. Lepidopteran families are written on their nodes where they separate from the

376 rest of the clades. Bootstrap values for the nodes are given below nodes, Baysian values are given

377 above.

378

379 Effect of HR-like necrosis on Pieris egg survival on different Brassicaceae plants

380 First, we also monitored egg survival of the abundant (in the Netherlands) Pieris species (both $P$.

381 napi and P. rapae) under natural field conditions. Egg survival was $40 \%$ lower when eggs induced

382 HR-like necrosis compared to survival of eggs that did not induce a leaf necrosis (GLM: $\chi^{2}=11.02$,

$383 \mathrm{df}=1, \mathrm{P}<0.001$, Fig. 5a). As not all eggs on a given plant elicited a necrosis, the fraction of eggs

384 eliciting HR-like necrosis was tested as well.

385 Second, we tested egg survival on three highly responding plant species from the first screening

386 under greenhouse conditions. HR-like necrosis significantly lowered the survival of singly laid $P$.

387 brassicae eggs on all three plant species (GLM: $\chi^{2}=38.41, \mathrm{df}=1, P<0.001$, fig. 5b). Plant species

388 alone significantly affected egg survival (GLM: $\left.\chi^{2}=6.38, \mathrm{df}=2, P=0.04\right)$, while the interaction

$389 \operatorname{did} \operatorname{not}\left(\mathrm{GLM}: \chi^{2}=3.25, \mathrm{df}=2, P=0.20\right)$. On $C$. hispanica plants egg survival was significantly

390 lower than on B. napus plants (pairwise MWU: $P=0.006$, Fig. 5b). 

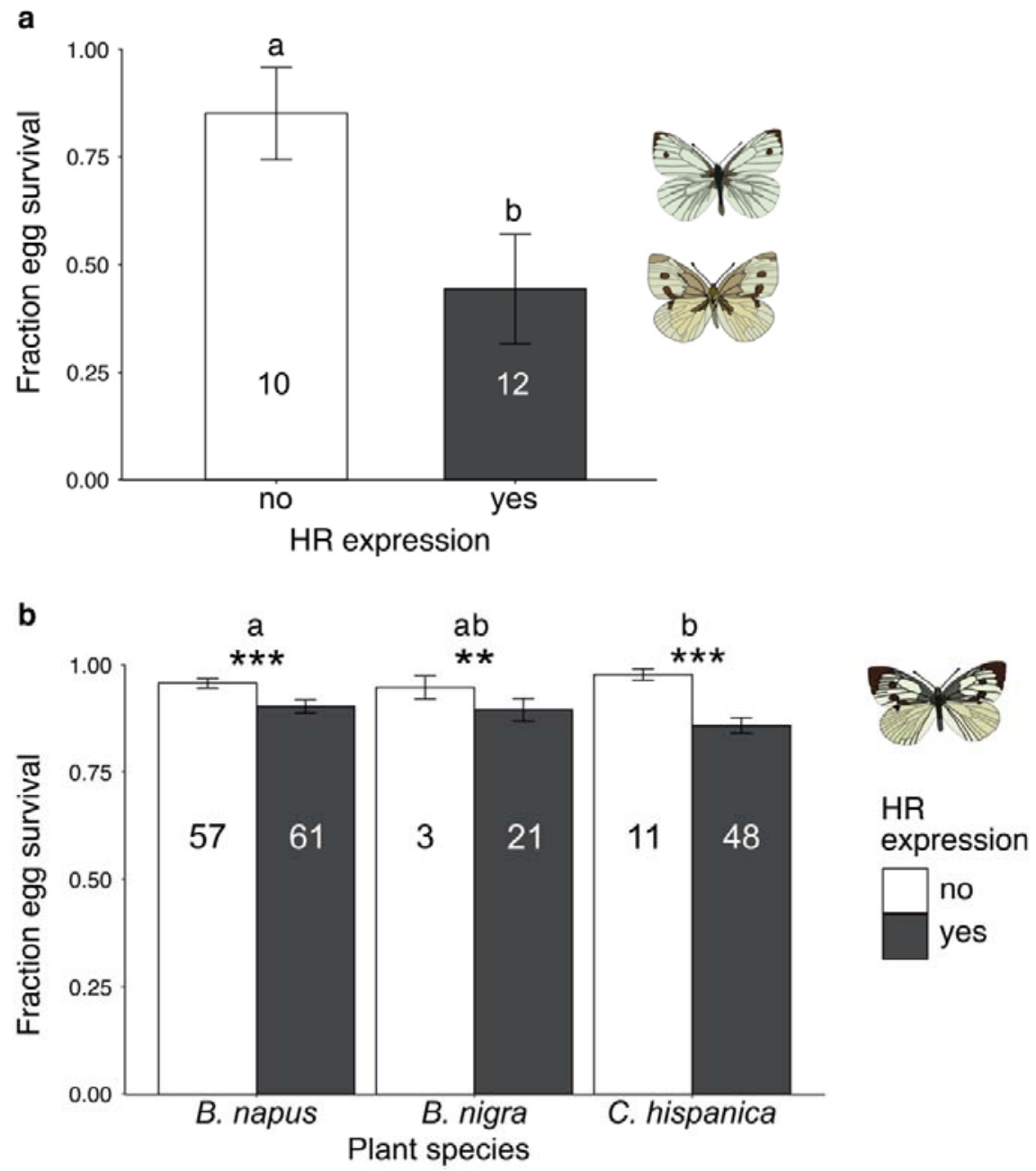

392 Figure 5: Survival rates of singly laid $P$. brassicae, $P$. rapae or $P$. napi eggs and effect of expression of HR-like necrosis on different plant species. A) Survey of $P$. napi and $P$. rapae eggs on B. nigra plants located near the river Rhine in Wageningen. One to 13 eggs were sampled per

395 plant, total number of collected eggs $n=96$. Fraction of survival depending on the expression of 396 HR-like necrosis by the plant. If the plant expressed HR-like necrosis under at least one egg it was 397 counted as HR-expressing 'yes'. Different letters indicate significant differences (GLM: $P<0.001$ ). 398 Numbers in bars indicate the number of plants surveyed within each category. B) Effect of HR-like 399 necrosis on survival rates (mean \pm SE) of singly laid $P$. brassicae eggs on B. napus, B. nigra and $C$. 400 hispanica. Asterisks indicate differences in egg survival between plants expressing HR-like necrosis 401 and non-HR within a plant species. Different letters indicate significant differences in egg survival 
402 between plant species, without taking HR-like necrosis into account. ns: not significant, **: P <

$403 \quad 0.01, * * *: \mathrm{P}<0.001$. (GLM).

404

405 Discussion

406 Pierid butterflies and their brassicaceous host plants are a fascinating model system of co-

407 evolutionary interactions; research so far has explored its evolutionary and genetic basis by

408 focusing on the diversifying selection on plant chemical defences, i.e. glucosinolates, and insect

409 NSP detoxification genes ${ }^{9,37,52}$. Here, we attempt for the first time to map the phylogenetic history

410 of an egg-induced plant defence trait and its reciprocal co-occurrence in the herbivore clade. We

411 show that pierid egg-induced HR-like necrosis evolved in two clades within the Brassicales. Half of

412 the tested plant species from the Brassiceae tribe in lineage II express strong HR-like necrosis to

413 egg wash. Moreover, all tested Aethionema species, the sister clade to the core Brassicaceae,

414 expressed leaf necrosis. Of the Brassica and Crambe plants (tribe Brassiceae) that were tested, the

415 HR-like necrosis lowered egg survival both under natural and greenhouse conditions. Furthermore,

416 we showed for the first time that only egg wash of Pieris butterflies and A. cardamines, specialist

417 feeders on the Brassicaceae, elicit a strong HR-like necrosis on B. nigra. While Colias spp. and G.

418 rhamni elicited a chlorotic response similar to that of Solanum dulcamara to Spodoptera eggs ${ }^{53}$.

419 Our results demonstrate that the egg-induced HR-like necrosis evolved as a new trait at least twice

420 in the Brassicales, but also show that plants specifically evolved this trait to lower egg survival of

421 those pierid species that evolved effective glucosinolate detoxification mechanisms.

423 Four out of eight tested Brassiceae species, as well as two tested Aethionema species showed

424 consistent HR-like necrosis to Pieris egg wash in at least one of the genotypes tested. In other plant

425 species, occasionally a single plant showed a light HR-like necrosis. Likely, those plants are false

426 positives, as some plants expressed a light necrosis to control (buffer) wash as well. Alternatively, it 
427 could be a general perception response of insect eggs as described for $A$. thaliana ${ }^{54}$. In the latter

428 species it was shown that a lectin receptor kinase, LecRK-I.8, might be involved in early perception

429 of eggs from two widely divergent species, P. brassicae and Spodoptera littoralis. The ancient

430 genome triplication event in the Brassiceae tribe might have facilitated the evolution of the HR-like

431 necrosis to eggs in this group by increasing the number of resistance genes underlying the trait.

432 Work is underway to identify the genes, which will contribute to a better understanding on the

433 evolution of HR-like necrosis. It is unlikely that the triplication event is the only factor involved in

434 the evolution of HR-like, because Aethionema plants respond to Pieris eggs with necrosis as well.

435 Aethionema species tested here are annuals that occur in dry habitats during a very short time of the

436 year ${ }^{55}$. Interestingly, most tested Brassiceae plants and Aethionema are host plants for different

437 Pieris butterflies. Both P. rapae and P. napi eggs are abundant in nature on B. nigra and its close

438 relatives like Sinapis arvensis ${ }^{17,19,55,56}$. Pieris ergane is described to feed on several Aethionema

439 species in their south eastern European habitat ${ }^{57}$.

441 Not all tested plant species within the Brassiceae tribe within Lineage II expressed HR-like

442 necrosis. This could be because we only selected non-responsive genotypes of these plant species or

443 genus. For example, Sinapis alba, did not show HR-like necrosis. However, previous work on the

444 close relative $S$. arvensis showed that eggs of $P$. rapae and $P$. brassicae strongly induced HR-like

445 necrosis $^{39}$. This means that that in some genera there is trait variation between species.

446 Alternatively, some plant species might have lost the ability to express HR-like necrosis. Those

447 plants could be less frequently used as host plants for pierid butterflies e.g. because of a

448 phenological mismatch between the plant species and its potential specialist herbivores, as e.g. in

449 the case of $A$. thaliana $^{58}$. In central Europe, A. thaliana is usually not attacked by pierid butterflies,

450 as it is rather small and usually completes its life-cycle before caterpillars could develop on the

451 plant $^{58}$. Notably, A. cardamines was observed to deposit eggs on A. thaliana in North Sweden 
452 where both life cycles briefly overlap ${ }^{59}$. Yet, Pieris eggs have not been reported to induce a leaf

453 necrosis lowering Pieris egg survival on different genotypes of A. thaliana including some Swedish

454 accessions $^{39,40,60}$, neither did we observe a visible necrosis on the tested genotype (Col-0) in our

455 experiments when using $P$. brassicae egg wash.

457 Strong induction of HR-like necrosis seems to be highly specific to Pieris butterfly species

458 belonging to the Pierinae clade and feeding on hosts belonging to the Brassiceae clade.

459 Interestingly, another Pierinae species, A. cardamines, induced HR-like but feeds on hosts

460 belonging to lineage I of the Brassicaceae (e.g. Cardamine sp. ${ }^{9}$ ). In the latter lineage we did not find

461 species responding with HR-like necrosis. When collecting A. cardamines eggs from the

462 inflorescence of Cardamine spp. we did not observe any HR-like necrosis (N.E. Fatouros, personal

463 observation). Wash from eggs of species from the non-brassicaceous Coliadinae subfamily, Colias

464 spp. and G. rhamni and the nymphalid A. io did not elicit HR-like necrosis. This suggests that the

465 elicitor for HR-like necrosis is specific for Pierinae butterflies that evolved with Brassicaceae plant

466 species rather than a general molecule present in butterfly eggs. Testing more pierid species from

467 different clades and host plant families is needed to confirm this hypothesis. So far, we also do not

468 know if slight differences of HR-like necrosis elicitation between different Pieris species is caused

469 by quantitative differences of the elicitor(s), or by changes in the chemical composition of the

470 elicitor(s). Currently, we are analysing the chemical composition of the egg wash from the different

471 butterfly species to identify the compounds inducing HR-like necrosis.

472

473 Previous work has shown that the NSP glucosinolate detoxification gene was a key innovation in

474 the ancestral Pierinae enabling them to shift host plant from Fabaceae to Brassicaceae ${ }^{9,37}$. A recent

475 study revealed another intriguing counter-adaptation to NSP genes: the speciose genus Erysimum

476 has recently gained a novel type of chemical defences, the toxic cardenolides. So far, no known 
477 specific adaptations to cardenolides have evolved in insect herbivores, including the Pieridae ${ }^{61}$. On

478 the other hand, pierid butterflies may already have found ways to counter-adapt to the egg-killing

479 HR-like necrosis. Clustered eggs of P. brassicae were shown to negate the egg-killing effect of the

480 HR-like necrosis ${ }^{18}$. While other advantages of egg clustering have been proposed before ${ }^{62}$, it clearly

481 is helpful in dealing with HR-like necrosis. Although the direct mechanisms of how clustering can

482 protect against egg-killing HR-like necrosis are unknown, it has been shown that desiccation can be

483 slowed down by clustering eggs ${ }^{18,63}$. This might be mitigated by the reduced egg surface area

484 exposed to the environment, compared with single eggs. Other pierid butterflies like $A$.

485 cardamines $^{64}$, P. mannii and P. napi have been observed to deposit their eggs near or on

486 inflorescence stems of their host plants (N.E. Fatouros, personal observation).

488 In conclusion, our findings demonstrate that various Brassicaceae plants can mount defences

489 against insect eggs and that these might be under similar selective pressures as plant defences

490 against feeding insects. A coevolutionary arms-race between Pieris butterfly eggs and plant species

491 within the Brassiceae clade as well as species within the sister clade Aethionema is likely to have

492 occurred. These plants make use of necrotic lesions to lower egg survival and might just have

493 evolved a new mechanism, possibly hijacked from disease resistances, to combat specialist

494 herbivores adapted to their host plants' toxins. Being a very early, premeditated defence, the

495 mechanism of HR-like necrosis is currently studied as a novel defensive trait to improve resistance

496 of Brassica crops against Pieris pests.

497

498

\section{Acknowledgements}

500 We thank the employees of Unifarm (Wageningen University and Research) for rearing and caring

501 of the experimental plants used in the experiment. We are thankful to Pieter Rouweler, André 
502 Gidding, Frans van Aggelen and Patrick Verbaarschot for rearing the Dutch Pieris butterflies used

503 in the experiment. Centre for Genetic Resources, the Netherlands, the Leibniz-Institut für

504 Pflanzengenetik und Kulturpflanzenforschung and BMAP consortium are thanked for the seeds.

505 Furthermore, we thank Prof. Miltos Tsiantis from the Department of Comparative Development and

506 Genetics, Max Planck Institute for Plant Breeding Research for kindly providing C. hirsuta seeds,

507 used as host plants for A. cardamines. This research has been made possible by funding of the

508 Netherlands Organisation for Scientific Research (NWO) to N.E.F. (NWO/ALW VIDI 14854 and

509 connected Aspasia).

\section{References}

5121 Ehrlich, P. R. \& Raven, P. H. Butterflies and plants: A study in coevolution. Evolution 18,

513 586-608 (1964).

5142 Becerra, J. X. Macroevolutionary and geographical intensification of chemical defense in

515 plants driven by insect herbivore selection pressure. Current Opinion in Insect Science $\mathbf{8}$,

$516 \quad 15-21(2015)$.

5173 Swain, T. Secondary compounds as protective agents. Annual Review of Plant Physiology

518 28, 479-501 (1977).

5194 Berenbaum, M. Coumarins and caterpillars: A case for coevolution. Evolution 37, 163-179

$520 \quad$ (1983).

5215 Després, L., David, J. P. \& Gallet, C. The evolutionary ecology of insect resistance to plant

522

523

524

525

526

527

528

529

530 Després, L., David, J. P. \& Gallet, C. The evolutionary ecology of in
chemicals. Trends in Ecology and Evolution 22, 298-307 (2007).

6 Cohen, J. A. Differences and similarities in cardenolide contents of queen and monarch butterflies in Florida and their ecological and evolutionary implications. Journal of Chemical Ecology 11, 85-1038 (1985).

7 Malcolm, S. B. \& Brower, L. P. Evolutionary and ecological implications of cardenolide sequestration in the monarch butterfly. Experientia 45, 284-295 (1989).

8 Heidel-Fischer, H. M. \& Vogel, H. Molecular mechanisms of insect adaptation to plant secondary compounds. Current Opinion in Insect Science 8, 8-14 (2015). Wheat, C. W. et al. The genetic basis of a plant-insect coevolutionary key innovation. Proceedings of the National Academy of Sciences of the United States of America 104, 20427-20431 (2007).

10 Wittstock, U. et al. Successful herbivore attack due to metabolic diversion of a plant chemical defense. Proceedings of the National Academy of Sciences of the United States of America 101, 4859-4864 (2004).

537 Hilker, M. \& Fatouros, N. E. Resisting the onset of herbivore attack: plants perceive and respond to insect eggs. Current Opinion in Plant Biology 32, 9-16 (2016). killing plant defenses for sustainable crop protection. Ecology and Evolution 6, 6906-6918 (2016). 
$541 \quad 13$ Seino, Y., Suzuki, Y. \& Sogawa, K. An ovicidal substance produced by rice plants in

542

543

544

545

546

547

548

549

550

551

552

553

554

555

556

557

558

559

560

561

562

563

564

565

566

567

568

569

570

571

572

573

574

575

576

577

578

579

580

581

582

583

584

585

586

587

588

589

response to oviposition by the whitebacked planthopper, Sogatella furcifera (Horvath)

(Homoptera: Delphacidae). Applied Entomology and Zoology 31, 467-473 (1996).

14 Doss, R. P. et al. Bruchins: Insect-derived plant regulators that stimulate neoplasm formation. Proceedings of the National Academy of Sciences of the United States of America 97, 6218-6223 (2000).

15 Petzold-Maxwell, J., Wong, S., Arellano, C. \& Gould, F. Host plant direct defence against eggs of its specialist herbivore, Heliothis subflexa. Ecological Entomology 36, 700-708 (2011).

16 Balbyshev, N. F. \& Lorenzen, J. H. Hypersensitivity and egg drop: A novel mechanism of host plant resistance to Colorado potato beetle (Coleoptera: Chrysomelidae). Journal of Economic Entomology 90, 652-657 (1997).

17 Fatouros, N. E. et al. Synergistic effects of direct and indirect defences on herbivore egg survival in a wild crucifer. Proceedings of the Royal Society of London B 281, 20141254 (2014).

18 Griese, E., Dicke, M., Hilker, M. \& Fatouros, N. E. Plant response to butterfly eggs: inducibility, severity and success of egg-killing leaf necrosis depends on plant genotype and egg clustering. Scientific Reports 7, 7316 (2017).

19 Shapiro, A. M. \& DeVay, J. E. Hypersensitivity reaction of Brassica nigra L (Cruciferae) kills eggs of Pieris butterflies (Lepidoptera, Pieridae). Oecologia 71, 631-632 (1987).

20 Bittner, N., Trauer-Kizilelma, U. \& Hilker, M. Early plant defence against insect attack: involvement of reactive oxygen species in plant responses to insect egg deposition. Planta 245, 993-1007 (2017).

21 Yang, Y. et al. Quantitative trait loci identification, fine mapping and gene expression profiling for ovicidal response to whitebacked planthopper (Sogatella furcifera Horvath) in rice (Oryza sativa L.). BMC Plant Biology 14, 145 (2014).

22 Garza, R., Vera, J., Cardona, C., Barcenas, N. \& Singh, S. P. Hypersensitive response of beans to Apion godmani (Coleoptera: Curculionidae). Journal of Economic Entomology 94, 958-962 (2001).

23 Pashalidou, F. G., Fatouros, N. E., Van Loon, J. J. A., Dicke, M. \& Gols, R. Plant-mediated effects of butterfly egg deposition on subsequent caterpillar and pupal development, across different species of wild Brassicaceae. Ecological Entomology 40, 444-450 (2015).

24 Al-Shehbaz, I. A. A generic and tribal synopsis of the Brassicaceae (Cruciferae). Taxon 61, 931-954 (2012).

25 Guo, X. et al. Plastome phylogeny and early diversification of Brassicaceae. BMC Genomics 18, 176 (2017).

26 Huang, C.-H. et al. Resolution of Brassicaceae phylogeny using nuclear genes uncovers nested radiations and supports convergent morphological evolution. Molecular Biology and Evolution 33, 394-412 (2015).

27 Beilstein, M. A., Al-Shehbaz, I. A. \& Kellogg, E. A. Brassicaceae phylogeny and trichome evolution. American Journal of Botany 93, 607-619 (2006).

28 Beilstein, M. A., Al-Shehbaz, I. A., Mathews, S. \& Kellogg, E. A. Brassicaceae phylogeny inferred from phytochrome $\mathrm{A}$ and $\mathrm{ndhF}$ sequence data: tribes and trichomes revisited. American Journal of Botany 95, 1307-1327 (2008).

29 Hall, J. C., Sytsma, K. J. \& Iltis, H. H. Phylogeny of Capparaceae and Brassicaceae based on chloroplast sequence data. American Journal of Botany 89, 1826-1842 (2002).

30 Graser, G., Schneider, B., Oldham, N. J. \& Gershenzon, J. The methionine chain elongation pathway in the biosynthesis of glucosinolates in Eruca sativa (Brassicaceae). Archives of Biochemistry and Biophysics 378, 411-419 (2000). 
$59031 \quad$ Rask, L. et al. Myrosinase: gene family evolution and herbivore defense in Brassicaceae.

591

592

593

594

595

596

597

598

599

600

601

602

603

604

605

606

607

608

609

610

611

612

613

614

615

616

617

618

619

620

621

622

623

624

625

626

627

628

629

630

631

632

633

634

635

636

637

638

Plant Molecular Biology 42, 93-114 (2000).

32 Windsor, A. J. et al. Geographic and evolutionary diversification of glucosinolates among near relatives of Arabidopsis thaliana (Brassicaceae). Phytochemistry 66, 1321-1333 (2005).

33 Xue, J., Lenman, M., Falk, A. \& Rask, L. The glucosinolate-degrading enzyme myrosinase in Brassicaceae is encoded by a gene family. Plant Molecular Biology 18, 387-398 (1992).

34 Erb, M. \& Robert, C. A. M. Sequestration of plant secondary metabolites by insect herbivores: molecular mechanisms and ecological consequences. Current Opinion in Insect Science 14, 8-11 (2016).

35 Heidel-Fischer, H. M. et al. An insect counteradaptation against host plant defenses evolved through concerted neofunctionalization. Molecular Biology and Evolution 36, 930-941 (2019).

36 Winde, I. \& Wittstock, U. Insect herbivore counteradaptations to the plant glucosinolatemyrosinase system. Phytochemistry 72, 1566-1575 (2011).

37 Edger, P. P. et al. The butterfly plant arms-race escalated by gene and genome duplications. Proceedings of the National Academy of Sciences of the United States of America 201503926 (2015).

38 Braby, M. F. \& Trueman, J. W. H. Evolution of larval host plant associations and adaptive radiation in pierid butterflies. Journal of Evolutionary Ecology 19, 1677-1690 (2006).

39 Griese, E. et al. Plant responses to butterfly oviposition partly explain preferenceperformance relationships on different brassicaceous species. bioRxiv, 706044 (2019).

40 Little, D., Gouhier-Darimont, C., Bruessow, F. \& Reymond, P. Oviposition by pierid butterflies triggers defense responses in Arabidopsis. Plant Physiology 143, 784-800 (2007).

41 Reymond, P. J. P. Perception, signaling and molecular basis of oviposition-mediated plant responses. 238, 247-258 (2013).

42 Ratnasingham, S. \& Hebert, P. D. N. BOLD: The Barcode of Life Data System (http://www.barcodinglife.org). Molecular Ecology Notes 7, 355-364 (2007).

43 Miller, M. A., Pfeiffer, W. \& Schwartz, T. in 2010 Gateway Computing Environments Workshop (GCE). 1-8.

44 Stamatakis, A. RAxML version 8: a tool for phylogenetic analysis and post-analysis of large phylogenies. Bioinformatics 30, 1312-1313 (2014).

45 Chernomor, O., Minh, B. Q. \& von Haeseler, A. Terrace aware data structure for phylogenomic inference from supermatrices. Systematic Biology 65, 997-1008 (2016).

46 Hoang, D. T., Vinh, L. S., Chernomor, O., Minh, B. Q. \& von Haeseler, A. UFBoot2: Improving the ultrafast bootstrap approximation. Molecular Biology and Evolution 35, 518522 (2017).

47 Trifinopoulos, J., Nguyen, L.-T., Minh, B. Q. \& von Haeseler, A. W-IQ-TREE: a fast online phylogenetic tool for maximum likelihood analysis. Nucleic Acids Research 44, W232W235 (2016).

48 Braby, M. F., Vila, R. \& Pierce, N. E. Molecular phylogeny and systematics of the Pieridae (Lepidoptera: Papilionoidea): Higher classification and biogeography. Zoological Journal of the Linnean Society 147, 239-275 (2006).

49 Ronquist, F. et al. MrBayes 3.2: Efficient bayesian phylogenetic inference and model choice across a large model space. Systematic Biology 61, 539-542 (2012).

50 Tracer v1.5.0 (2009).

51 R: A Language and Environment for Statistical Computing v. 3.3.2 "Sincere Pumpkin Patch" (R Foundation for Statistical Computing, Vienna, Austria, 2016).

52 Nallu, S. et al. The molecular genetic basis of herbivory between butterflies and their host plants. Nature Ecology and Evolution 2, 1418-1427 (2018). 
648

649

650

651

652

653

654

655

656

657

658

659

660

661

662

663

664

665

666

667

668

669

670

671

672

673

674

675

676
53 Geuss, D., Stelzer, S., Lortzing, T. \& Steppuhn, A. Solanum dulcamara's response to eggs of an insect herbivore comprises ovicidal hydrogen peroxide production. Plant, Cell and Environment 40, 2663-2677 (2017).

54 Mohammadin, S. et al. Genome-wide nucleotide diversity and associations with geography, ploidy level and glucosinolate profiles in Aethionema arabicum (Brassicaceae). Plant Systematics and Evolution 304, 619-630 (2018).

55 Fei, M., Gols, R. \& Harvey, J. A. Seasonal phenology of interactions involving short-lived annual plants, a multivoltine herbivore and its endoparasitoid wasp. Journal of Animal Ecology 83, 234-244 (2014).

56 Friberg, M., Posledovich, D. \& Wiklund, C. J. O. Decoupling of female host plant preference and offspring performance in relative specialist and generalist butterflies. Oecologia 178, 1181-1192 (2015).

57 Tolman, T. \& Lewington, R. Collins butterfly guide: The most complete field guide to the butterflies of Britain and Europe. (Harper Collins, 2009).

58 Harvey, J. A., Witjes, L. M. A., Benkirane, M., Duyts, H. \& Wagenaar, R. J. P. E. Nutritional suitability and ecological relevance of Arabidopsis thaliana and Brassica oleracea as foodplants for the cabbage butterfly, Pieris rapae. Plant Ecology 189, 117-126 (2007).

59 Wiklund, C. \& Friberg, M. The evolutionary ecology of generalization: among-year variation in host plant use and offspring survival in a butterfly. Ecology 90, 3406-3417 (2009).

60 Vrolings, T. Intraspecific variation of Pieris rapae oviposition-induced plant defences in different natural accessions of Arabidopsis thaliana Master thesis, Wageningen University, (2014).

61 Züst, T. et al. Rapid and independent evolution of ancestral and novel defenses in a genus of toxic plants (Erysimum, Brassicaceae). BioRxiv 761569 (2019).

62 Courtney, S. P. The evolution of egg clustering by butterflies and other insects. The American Naturalist 123, 276-281 (1984).

63 Clark, B. R. \& Faeth, S. H. The evolution of egg clustering in butterflies: A test of the egg desiccation hypothesis. Evolutionary Ecology 12, 543-552, (1998).

64 Wiklund, C. \& Åhrberg, C. Host plants, nectar source plants, and habitat selection of males and females of Anthocharis cardamines (Lepidoptera). Oikos 31, 169-183 (1978). 